\title{
Clinical and Genetic Characteristics of Patients with Corticosterone Methyloxidase Deficiency Type 2: Novel Mutations in CYP11B2
}

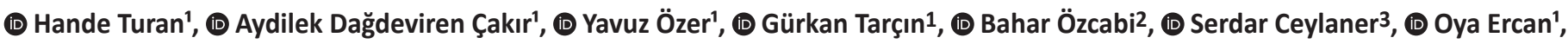 \\ (D) Saadet Olcay Evliyaoğlu' ${ }^{1}$ \\ 1istanbul University-Cerrahpaşa, Cerrahpaşa Faculty of Medicine, Department of Pediatric Endocrinology, Istanbul, Turkey \\ 2Zeynep Kamil Training and Research Hospital, Clinic of Pediatric Endocrinology, Istanbul, Turkey \\ 3 Intergen Genetic Diagnosis Center, Medical Genetics, Ankara, Turkey
}

\begin{abstract}
What is already known on this topic?
Corticosterone methyloxidase deficiency type 2 is an autosomal recessive disorder which presents with salt loss and failure to thrive in early childhood. It is caused by inactivating mutations of CYP11B2. To date, approximately 56 mutations have been identified in the CYP11B2 gene.
\end{abstract}

\section{What this study adds?}

We describe four Turkish patients from two families who have clinical and hormonal features compatible with corticosterone methyloxidase deficiency and had inherited novel CYP11B2 mutations.

\begin{abstract}
Corticosterone methyloxidase deficiency type 2 is an autosomal recessive disorder presenting with salt loss and failure to thrive in early childhood and is caused by inactivating mutations of the CYP11B2 gene. Herein, we describe four Turkish patients from two families who had clinical and hormonal features compatible with corticosterone methyloxidase deficiency and all had inherited novel CYP11B2 variants. All of the patients presented with vomiting, failure to thrive and severe dehydration, except one patient with only failure to thrive. Biochemical studies showed hyponatremia, hyperkalemia and acidosis. All patients had normal cortisol response to adrenocorticotropic hormone stimulation test and had elevated plasma renin activity with low aldosterone levels. Three patients from the same family were found to harbor a novel homozygous variant c.1175T > C (p.Leu392Pro) and a known homozygous variant c.788T > A (p.Ile263Asn) in the CYP11B2 gene. The fourth patient had a novel homozygous variant c.666_667delCT (p.Phe223ProfsTer35) in the CYP11B2 gene which caused a frame shift, forming a stop codon. Corticosterone methyloxidase deficiency should be considered as a differential diagnosis in patients presenting with hyponatremia, hyperkalemia and growth retardation, and it should not be forgotten that this condition is life-threatening if untreated. Genetic analyses are helpful in diagnosis of the patients and their relatives. Family screening is important for an early diagnosis and treatment. In our cases, previously unreported novel variants were identified which are likely to be associated with the disease.
\end{abstract}

Keywords: Aldosterone synthase deficiency, salt wasting, CYP11B2 gene, corticosterone methyloxidase type 2, failure to thrive

\section{Introduction}

Aldosterone is a steroid hormone synthesized by corticosterone methyloxidase (CMO) and secreted from the zona glomerulosa of the adrenal cortex. CMO catalyzes the final three steps in aldosterone synthesis (11 $\beta$-hydroxylase, 18-hydroxylase, and, lastly, 18-methyloxidase), as the most important steps in aldosterone biosynthesis, which takes place only in the zona glomerulosa $(1,2)$. In humans, two $11 \beta$-hydroxylase isoenzymes are encoded by two genes
Address for Correspondence: Hande Turan MD, İstanbul University-Cerrahpaşa, Cerrahpaşa Faculty of Medicine, Department of Pediatric Endocrinology, İstanbul, Turkey

Phone: +90 5059113735 E-mail: dr.handeerdogan@gmail.com ORCID: orcid.org/0000-0003-0121-3756
Conflict of interest: None declared Received: 25.12.2019 Accepted: 09.06.2020

${ }^{\circ}$ Copyright 2021 by Turkish Pediatric Endocrinology and Diabetes Society

The Journal of Clinical Research in Pediatric Endocrinology published by Galenos Publishing House. 
located on the long arm of chromosome 8 (3). CYP11B1 expression is primarily controlled by adrenocorticotropic hormone $(\mathrm{ACTH})$, which acts through a specific G-proteincoupled receptor to increase levels of cyclic adenosine monophosphate. CYP11B2 is mainly regulated by angiotensin 2 and potassium. The promoter region of both genes is strikingly different, underlining the fact that both genes are differently regulated on the transcriptional level, leading to two dissimilar types of disease. Both types CMO type 1 deficiency (OMIM 203400) and CMO type 2 deficiency (OMIM 610600) have similar signs and symptoms but can be distinguished by laboratory testing. These conditions can be differentiated by the presence of insufficient or excessive 18-OH-corticosterone. In CMO 2 deficiency, despite high levels of 18-hydroxycorticosterone (18-OHB), aldosterone levels remain low or normal. These patients have a low ratio of corticosterone to 18-OHB (4).

CMO deficiency (CMOD) type 2 is a rare disorder with unknown prevalence. A particularly high population density of CMOD type 2 was identified in Iranian Jews from the city Isfahan (5), but the disease has been documented throughout Europe and North America $(6,7)$.

CMOD can cause nausea, vomiting, dehydration, low blood pressure, extreme tiredness (fatigue) and muscle weakness, associated with hyponatremia, hyperkalemia and metabolic acidosis. Severe cases of CMOD can result in seizures and coma. Affected infants often exhibit failure to thrive. The signs and symptoms of the disorder typically become milder or disappear by adulthood.

\section{Case Reports}

\section{Family 1}

\section{Family 1-1}

A six-month-old boy was admitted with salt loss and failure to thrive and moderate dehydration. He is the first child of consanguineous parents (Figure 1a), born with a birth weight of $2900 \mathrm{~g}$ and length of $50 \mathrm{~cm}$. Physical examination revealed growth retardation, cachectic appearance, and decreased subcutaneous adipose tissue. His height and weight standard deviation scores (SDS) were -1.64 and -2.16, respectively. External genital appearance was normal. He had no hyperpigmentation. Blood pressure was normal $\left(95^{\text {th }}\right.$ percentile $\left.=99 / 55 \mathrm{mmHg}\right)$ (Table 1$)$. He had hyponatremia and hyperkalemia despite elevated renin and normal aldosterone levels (Table 2). His plasma 18OHB level and 18-OHB to aldosterone ratio were increased (Table 2). All the other adrenal hormones including 17-hydroxyprogesterone (17-OH), androstenedione, total testosterone, (dehydroepiandrosterone-sulfate) and cortisol and his ACTH concentrations were within normal limits. He was diagnosed as isolated aldosterone deficiency and, thus, salt and fludrocortisone treatments were initiated. In his follow-up, his electrolytes and anthropometric measurements had normalized (height SDS -0.5, weight SDS 0.28). Genetic analysis revealed two different homozygous variants in the CYP11B2 (NM_000498.3) confirming the diagnosis of CMOD type 2 . The first variant changes thymine to adenine at nucleotide 788 (c.788T > A), resulting in an isoleucine-to-asparagine substitution at codon 263 (p.Ile263Asn) (8). The latter variant is novel and changes thymine to cytosine at nucleotide 1157 (c. $1157 \mathrm{~T}>\mathrm{C}$ ), resulting in a leucine to proline substitution at codon 392 (p.Leu392Pro). Consanguineous parents were heterozygous for both variants without any clinical findings. The variants NM_000498.3:c.1175T > C (p.Leu392Pro) and NM_000498.3:c.788T > A (p.Ile263Asn) were evaluated by American College of Medical Genetics (ACMG) criteria and classified as Variant of Unknown Significance.

\section{Family 1-2}

A two-year-old sibling of the first case was admitted with growth retardation. He was born at gestational week 38, with a weight of $3050 \mathrm{~g}$ and length of $50 \mathrm{~cm}$. His medical records revealed a history of hyponatremia and hyperkalemia at the age of thre months, which did not persist in later follow-up. At admission, although his electrolytes were within normal limits, his height and weight SDS were -1.99 and -2.14 , respectively (Table 1). Following fludrocortisone treatment, his growth characteristics normalized (height SDS 0.3, weight SDS -0.1). Genetic analysis revealed the same variant as that of his siblings.

\section{Family 1-3}

A three-month-old girl, sister of cases 1 and 2, was admitted due to poor weight gain. She was born at normal gestational age $(38+5$ weeks) with a birth weight of 2800 $g$ and length of $49 \mathrm{~cm}$. Her physical examination revealed normal female external genital development but decreased subcutaneous adipose tissue. Her height and weight SDSs were -2.82 and -1.62 , respectively (Table 1 ). Her blood pressure was $77 / 50 \mathrm{mmHg}$ ( $95^{\text {th }}$ percentile $98 / 53 \mathrm{mmHg}$ ). She had hyponatremia, mild hyperkalemia, increased renin, and normal aldosterone levels (Table 1). Following fludrocortisone treatment, adequate weight gain and height velocity were achieved and laboratory findings normalized (height SDS -0.4, weight SDS 0.08). Genetic analysis revealed the same variant as that of her siblings. 
Table 1. Clinical features of the patients, initial findings at presentation, laboratory results, genetic analyses and treatments

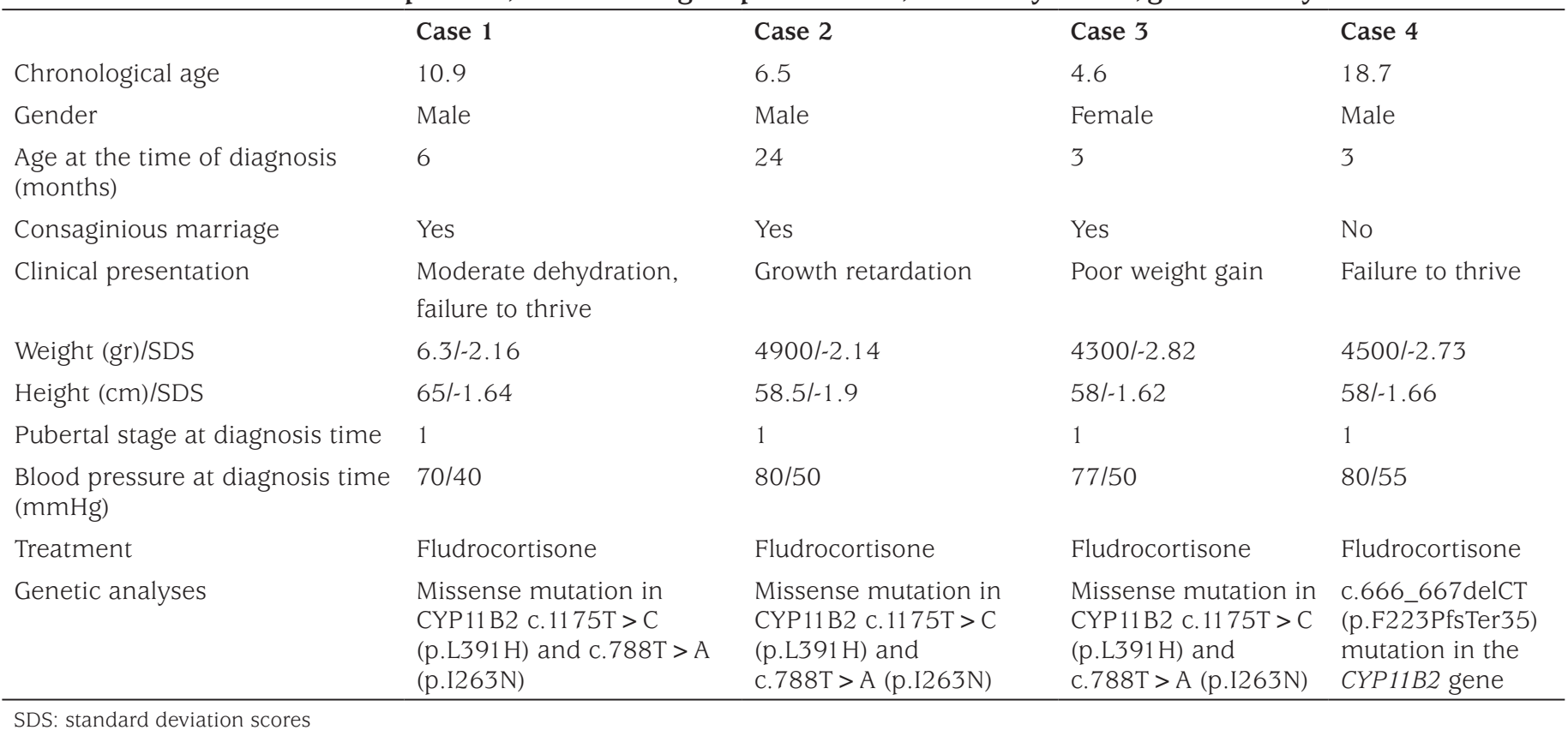

SDS. standard deviation scores

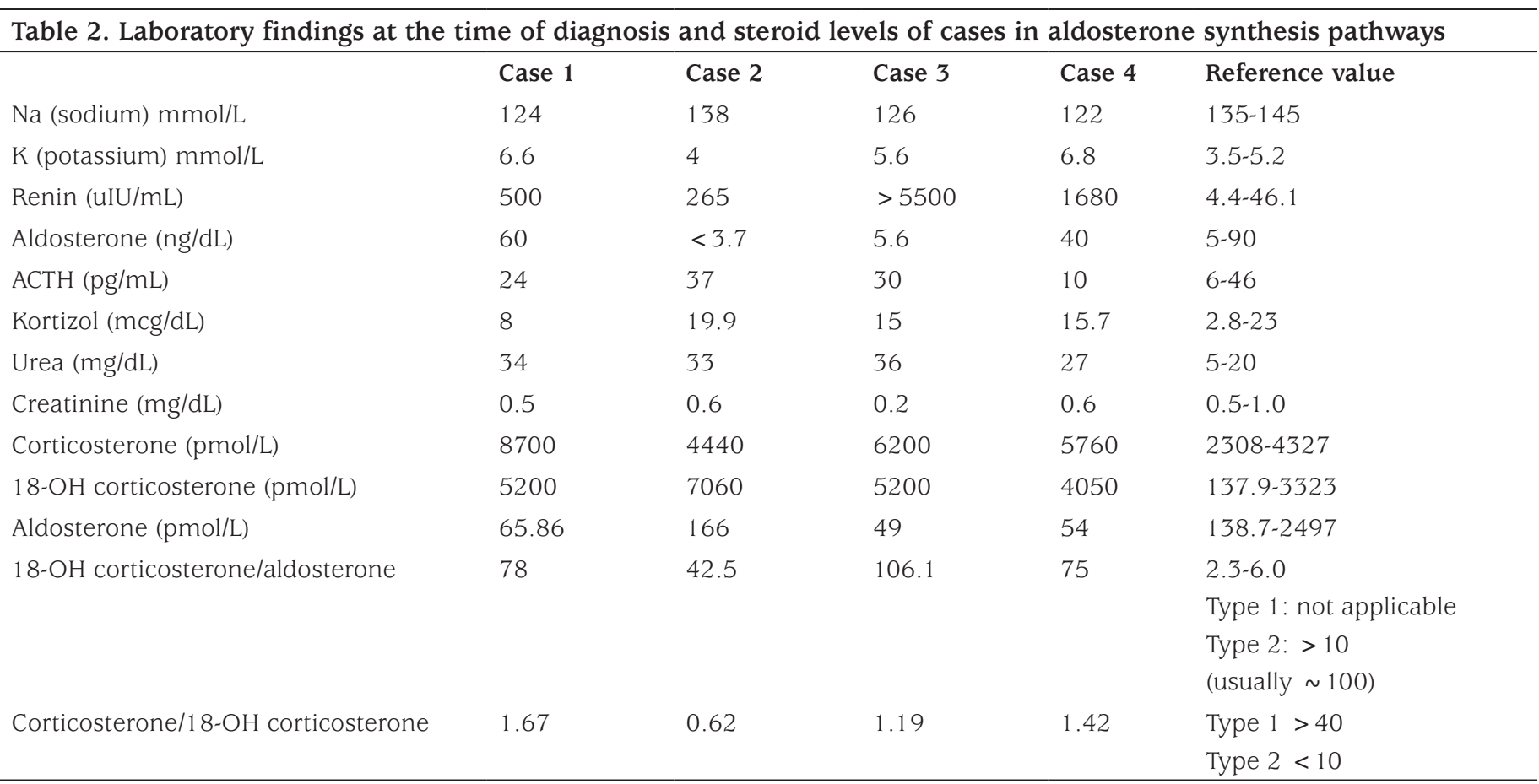

18-OH: 18-hydroxycorticosterone, ACTH: adrenocorticotropic hormone

\section{Family 2}

\section{Family 2-1}

A three-month-old-boy was brought to our clinic due to failure to thrive and vomiting. He was the first child of non-consanguineous healthy parents (Figure $1 \mathrm{~b}$ ), born with a weight of $3400 \mathrm{~g}$ and length of $50 \mathrm{~cm}$. On physical examination, mild dehydration and decreased subcutaneous adipose tissue were observed. His weight was $4500 \mathrm{~g}$ (SDS -2.73) and height was $58 \mathrm{~cm}$ (SDS -1.66) (Table 1). Laboratory investigation showed hyponatremia, hyperkalemia, increased plasma renin activity and low serum aldosterone concentration. Adrenal steroids and ACTH levels were within normal limits (Table 2). A diagnosis of isolated aldosterone deficiency was established and $0.1 \mathrm{mg}$ of fludrocortisone per day was initiated. A rapid weight gain, normalization 
of serum electrolytes, and normalization of plasma renin activity were achieved. As isolated aldosterone deficiency was the probable diagnosis, further investigations were not performed at that time.

In follow-up, he was reassessed at the age of 18 years and blood sample was sent for genetic analysis, which revealed a novel homozygous c.NM_000498.3:c.666_667delCT (p.F223PfsTer35) mutation in the CYP11B2 gene causing a frame shift and forming a stop codon, detected by Next Generation Sequencing. This variant, which was classified as pathogenic by ACMG criteria as it is a null variant, is predicted to result in replacing phenylalanine 223 with a proline, shifting the reading frame, and terminating at position Ter35 (p.Phe223ProfsTer35) (Figure 2). The patient is now 18.3 years old, receiving fludrocortisone treatment, and his height and weight SDS are 0.12 and 0.59 , respectively, and normal for age.

Genomic DNA was extracted from peripheral blood samples of the patients. Genetic analyzes were performed by next generation sequencing (Miseq, Illumina, SanDiego, USA) following manufacturers instructions.

\section{Discussion}

Aldosterone deficiency is very rare and is a life-threatening condition when not treated. Clinical presentation of CMOD varies by age. Since ions cross the placental barrier, despite congenital enzyme deficiency, there are no symptoms during fetal life (6). Infants with a mineralocorticoid synthesis defect may show signs of salt-wasting within the first few days or weeks of life. These findings may include vomiting, dehydration, hypovolemia, hyponatremia, hyperkalemia and metabolic acidosis. In children diagnosed in early childhood, growth failure, nutritional problems, mild dehydration and electrolyte disturbances are observed. Miao et al (9) reviewed 44 patients in the published literature and

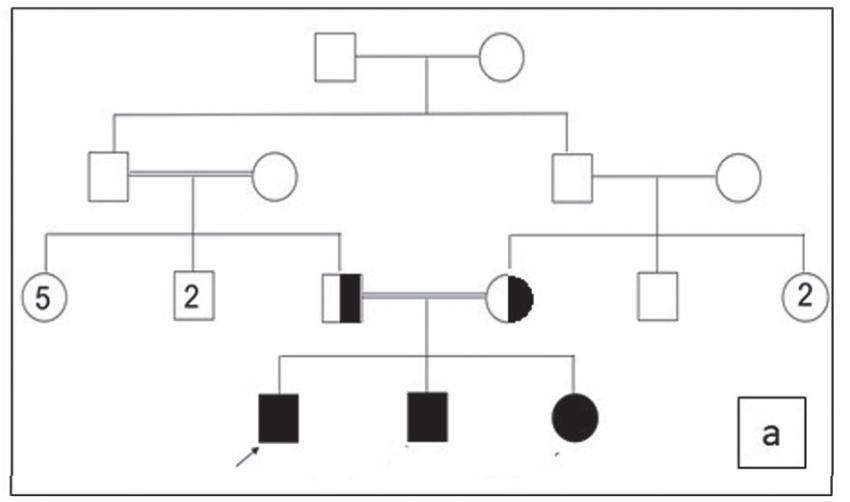

compared characteristics of cases with CMOD type 1 and type 2. Clinical features showed no significant difference in CMOD type 1 and 2. Failure to thrive, recurrent vomiting and dehydration were the most common symptoms in these patients. Although electrolyte disorder normalizes by the age of four years, growth retardation continues throughout childhood. Adults are generally asymptomatic, but they cannot tolerate severe salt loss. They are usually recognized in family screenings.

In the present study, age of diagnosis varied between three months and two years. Our cases have some clinical similarities and some differences to previously reported CMOD cases. Our three cases from the first family presented with vomiting, severe dehydration, hyponatremia and hyperkalemia, and one case, whose brother was diagnosed previously, was asymptomatic and presented only with growth retardation. Almost all patients, as in our cases, clinically improve with age, even if clinical severity among individuals may vary widely.

Mineralocorticoid deficiency causes hyponatremia and hyperkalemia by causing excessive sodium excretion and potassium retention in the renal distal tubule and cortical collection channel. In untreated infants with CMOD, serum sodium level is generally between $120-130 \mathrm{mmol} / \mathrm{L}$ and serum potassium level is between $6.0-8.5 \mathrm{mmol} / \mathrm{L} \mathrm{(10)}$. In accordance with the literature, in our patients, initial sodium and potassium levels were between 122-126 mmol/L and 5.6-7 mmol/L, respectively. All of our cases had high plasma renin activity and normal aldosterone levels (Table 2). Plasma renin activity is significantly increased in affected infants and young children (up to 100-fold normal) but can be normal in adults.

Two types of CMOD have been identified and these syndromes have the same clinical features but differ in the profiles of secreted steroids. Type 2 deficiency can be easily diagnosed by a marked increase in the ratio of $18-\mathrm{OHB}$

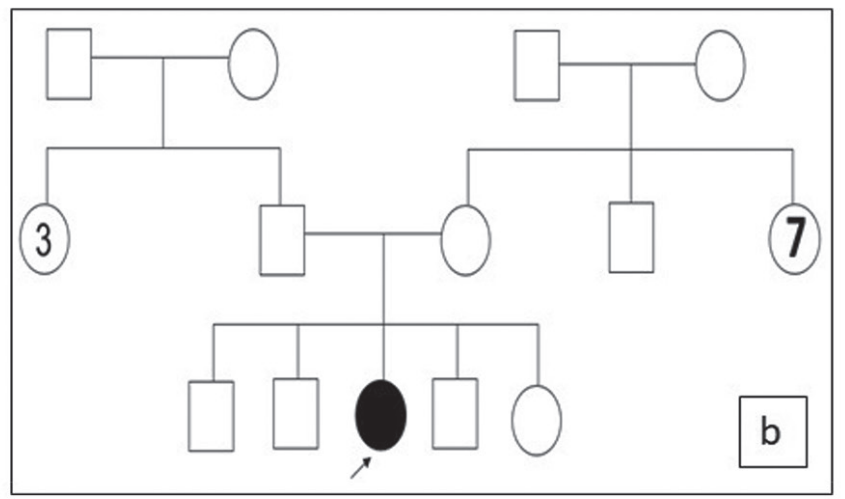

Figure 1. Pedigrees of families; a) Pedigree of family 1 ; b) Pedigree of family 2 


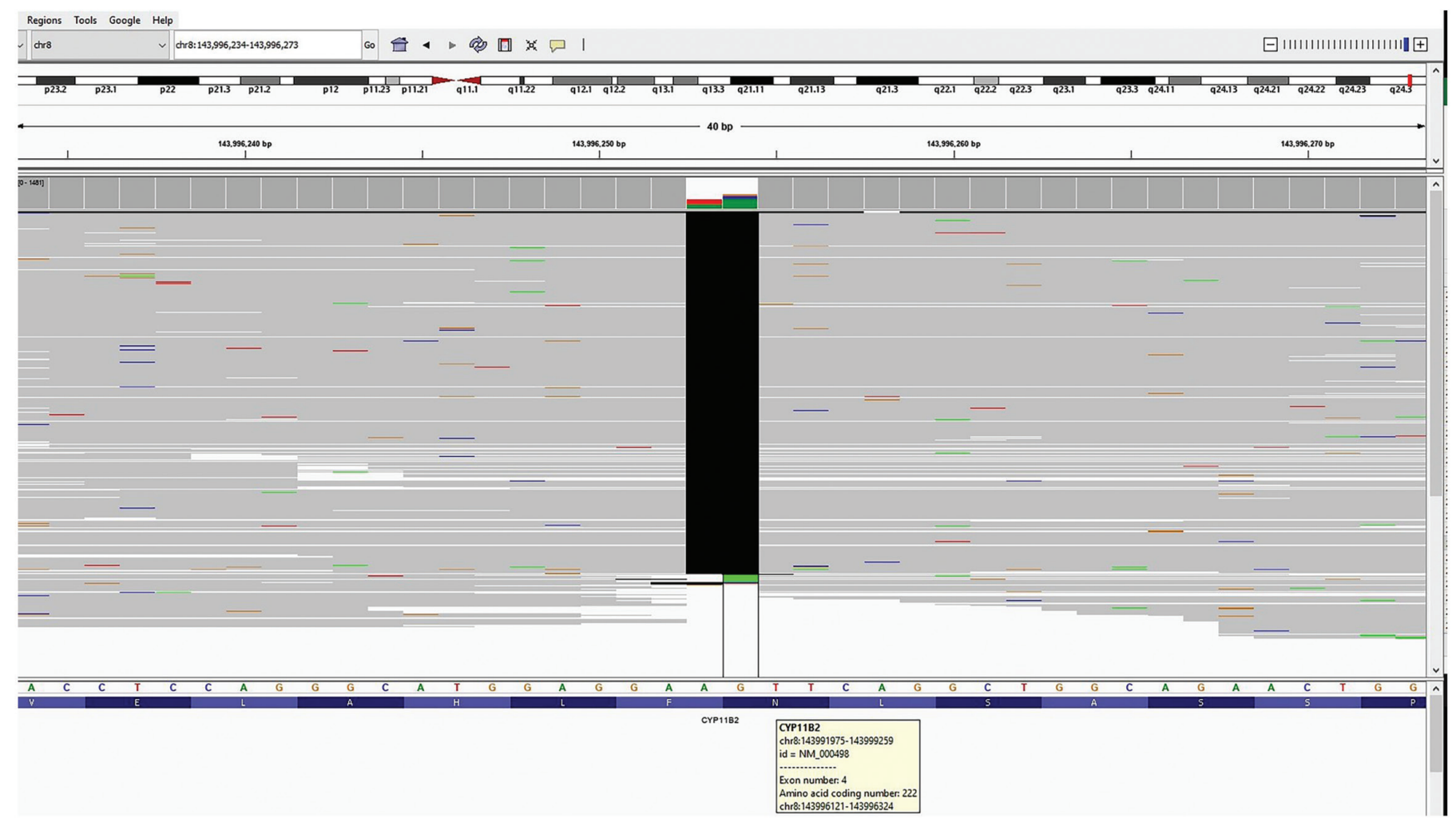

Figure 2. Image of genetic analysis of the patient "family 2-1"

to aldosterone in urine or serum (usually 100-fold). This ratio does not vary by age in affected individuals despite improving clinical features.

Steroid profiles of our patients are given in Table 2 and increased $18-\mathrm{OHB}$ to aldosterone ratios in urine or serum were consistent with type 2 CMOD. This ratio is not useful in the diagnosis of CMOD type 1 because very low levels of aldosterone make the ratio insignificant (11).

The most common disorder in patients presenting with hyponatremia, hyperkalemia and vomiting is congenital adrenal hyperplasia (CAH). $\mathrm{CAH}$ should be excluded because the defects of aldosterone synthesis are often seen as a part of cortisol production failure. Bizzarri et al (12) reported their ten-year-experience in infants presenting with hyponatremia and salt loss. Only 2 of 51 patients had aldosterone deficiency due to a CYP11B2 gene defect, and the majority $(37.5 \%$ ) was diagnosed with $\mathrm{CAH}$. The lack of ambiguous genitalia in our female patient, normal basal 17 $\mathrm{OH}$ progesterone levels or increased levels of renin and 18 $\mathrm{OH}$ progesterone helped to differentiated CMOD from $\mathrm{CAH}$ in our patients.

Another disorder to consider in the differential diagnosis is CMOD type 1. Patients with CMOD type 1 also present with similar clinical findings. High 18-OHB levels and 18-
OHB to aldosterone ratios differentiated our patients from CMOD type 1 , characterized by the presence of inadequate 18-OHB.

Pseudohypoaldosteronism (PHA) is another disease to be considered in the differential diagnosis. The underlying pathogenesis for PHA are unresponsive aldosterone receptor or overactive $\mathrm{Na}-\mathrm{Cl}$ co-transporter in the distal nephron. These patients do not improve on treatment with fludrocortisone due to resistance to aldosterone (8), but in our patients with CMOD, clinical findings improved with fludrocortisone treatment.

To date, approximately 56 mutations have been identified in the CYP11B2 gene. Primary hypoaldosteronism can be caused by different defects in CYP11B2, such as nonsense/ missense, splicing, regulatory and frame shift mutations, gross deletions and complex rearrangements (data from Human Gene Mutation Database) (13,14). Missense/ nonsense mutations constitute the largest proportion of these mutations (Approximately 70\%) (15). However, in this case report, we found two novel and one previously reported variant in the $C Y P 11 B 2$ gene.

Three siblings were homozygous for two substitution variants. A novel variant, C.1175T $>$ C (p.Leu392Pro) resulted in a leucine to proline substitution at codon 392. 
The other variant is another substitution CYP11B2 variant located in exon 4 . This variant changes thymine to adenine at nucleotide 788 (c.788T $>$ A), resulting in an isoleucine to asparagine substitution at codon 263. These variants were not detected in GnomAD exomes and GnomAD genomes databases. We also checked our own 2500 exome data and we could not find these variants. As the clinical picture of our patients clearly fits with the disorder, these variants were classified as "likely pathogenic". One of these two variants or both of them may be pathogenic. The other possibility is that these two variants may be pathogenic when present together on the same allel.

The c.788T > A (p.Ile263Asn) variant was first described in 2016 by Üstyol et al (16). This pathogenic variant has so far been reported only in Turkish patients supporting Turan et al (8) who suggested an ethnic specificity of the variant. As all cases carrying this variant were reported to have the clinical features of CMOD type 2, this variant is more likely to be pathological. So far, no functional enzymatic studies of this variant have been conducted, but the clinical presentation correlated well with previous studies. The parents of our three siblings are heterozygous for the same variants. In the genetic study of case 4 , a novel homozygous two base pair frame shift mutation (c.666_667delCT) was found in CYP11B2, resulting in a stop codon. Due to this premature stop codon this variant is very probably related to the disease. However, functional analysis of genes should still be performed to determine the functional outcome of the loss in gene product.

Clinical symptoms of different severity can be observed in patients with the same mutation. Twelve patients from eight families, reported in 1977, had the same mutation but there was a marked range in clinical severity, which varied from an asymptomatic state in adulthood to acute salt-wasting crisis in infancy, detected only by biochemical profile. So researchers concluded that individual differences in the degree of severity do not reflect the allele variant (5). Instead, they indicate the effects of other genetic loci or non-genetic factors (17).

Fludrocortisone replacement is necessary to correct the deficiency. The response to mineralocorticoid replacement and salt supplementation (dramatic catch-up growth, no further diarrhea or vomiting and normalized appetite) confirmed the diagnosis. Salt wasting, possibly due to insulin-like growth factor-1 suppression or reduced extracellular fluid volume and could be a factor leading to impaired growth (9).

Some studies suggest that mineralocorticoid therapy should be given for linear growth, despite normal serum electrolytes $(18,19)$. Clinical improvement in growth rate with mineralocorticoid therapy in reported cases with no ion deficits but growth failure, also support this view. This condition can be explained by chronic salt wasting. Prospective studies have shown poor linear growth when both rats and humans are fed sodium deficient diets. The $\mathrm{Na}-\mathrm{H}$ antiporter, present in many types of cell membranes, is an important mediator of cell growth and proliferation by its action in alkalinizing the cell interior (20).

Salt-wasting improves with aging and the majority of the cases can be asymptomatic in adulthood, even if not treated, with normal electrolyte levels $(20,21)$. There are some reasons why the mineralocorticoid requirements decrease with age. Firstly, mineralocorticoid receptors are poorly expressed in the renal epithelium of newborns and this increases with age. Secondly, newborn diets (breastfeeding) have low sodium content, and dietary sodium intake increases with age (9). Other reasons mentioned before including increased sodium reabsorption due to mature renal tubules and alternative pathway of mineralocorticoid biosynthesis (12). In follow-up, patients should be evaluated carefully because the need for treatment decreases with increasing age and keeping the same dosage of fludrocortisone may lead to hypokalemia and hypertension (18). Our patients continue to be treated but with reduced doses.

\section{Conclusion}

CMOD should be considered in the differential diagnosis in patients presenting with hyponatremia, hyperkalemia and growth retardation and it should not be forgotten that this condition is life-threatening if not treated. Genetic analyses are beneficial for diagnosis of the patients and other relatives at the risk of salt loss and failure to thrive. The same variants may result in a varying severity of clinical findings in different patients, even within the same family. Thus, family screening is important for early diagnosis and treatment.

\section{Ethics}

Informed Consent: We state that the subjects and their parents have given their written informed consent to publish their cases, in accordance with the Declaration of Helsinki.

Peer-review: Externally peer-reviewed.

\section{Authorship Contributions}

Concept: Hande Turan, Oya Ercan, Saadet Olcay Evliyaoğlu, Design: Hande Turan, Gürkan Tarçın, Oya Ercan, Saadet Olcay Evliyaoğlu, Data Collection or Processing: Hande Turan, Aydilek Dağdeviren Çakır, Yavuz Özer, Bahar Özcabi, 
Serdar Ceylaner, Oya Ercan, Saadet Olcay Evliyaoğlu, Analysis or Interpretation: Hande Turan, Saadet Olcay Evliyaoğlu, Serdar Ceylaner, Literature Search: Hande Turan, Aydilek Dağdeviren Çakır, Yavuz Özer, Gürkan Tarçın, Writing: Hande Turan, Gürkan Tarçın, Oya Ercan, Saadet Olcay Evliyaoğlu.

Financial Disclosure: The authors declared that this study received no financial support.

\section{References}

1. Curnow KM, Tusie-Luna MT, Pascoe L, Natarajan R, Gu JL, Nadler JL, White PC. The product of the CYP11 B2 gene is required for aldosterone biosynthesis in the human adrenal cortex. Mol Endocrinol 1991;5:15131522

2. Ogishima T, Shibata H, Shimada H, Mitani F, Suzuki H, Saruta T, Ishimura Y. Aldosterone synthase cytochrome P-450 expressed in the adrenals of patients with primary aldosteronism. J Biol Chem 1991;266:10731-10734

3. Chua SC, Szabo P, Vitek A, Grzeschik KH, John M, White PC. Cloning of CDNA encoding steroid 11 beta-hydroxylase (P450c11). Proc Natl Acad Sci U S A 1987;84:7193-7197.

4. Portrat-Doyen S, Tourniaire J, Richard O, Mulatero P, Aupetit-Faisant B, Curnow KM, Pascoe L, Morel Y. Isolated aldosterone synthase deficiency caused by simultaneous E198D and V386A mutations in the CYP11B2 gene. J Clin Endocrinol Metab 1998;83:4156-4161.

5. Katznelson D, Sack J, Kraiem Z, Lunenfeld B. Congenital hypoaldosteronism. Thirteen year follow-up in identical twins. Horm Res 1979;11:22-28.

6. Collinet E, Pelissier P, Richard O, Gay C, Pugeat M, Morel Y, Stephan JL. Déficit en aldostérone-synthase: 4 observations pédiatriques [Four cases of aldosterone synthase deficiency in childhood]. Arch Pediatr 2012;19:1191-1195. Epub 2012 Oct 10

7. Pascoe L, Curnow KM, Slutsker L, Rösler A, White PC. Mutations in the human CYP11B2 (aldosterone synthase) gene causing corticosterone methyloxidase II deficiency. Proc Natl Acad Sci USA 1992;89:49965000 .

8. Turan I, Kotan LD, Tastan M, Gurbuz F, Topaloglu AK, Yuksel B. Molecular genetic studies in a case series of isolated hypoaldosteronism due to biosynthesis defects or aldosterone resistance. Clin Endocrinol (Oxf) 2018;88:799-805. Epub 2018 Apr 18
9. Miao H, Yu Z, Lu L, Zhu H, Auchus RJ, Liu J, Jiang J, Pan H, Gong F, Chen S, Lu Z. Analysis of novel heterozygous mutations in the CYP11B2 gene causing congenital aldosterone synthase deficiency and literature review. Steroids 2019;150:108448. Epub 2019 Jul 11

10. White PC. Steroid 11 beta-hydroxylase deficiency and related disorders White PC. Steroid 11 beta-hydroxylase deficiency and related disorders. Endocrinol Metab Clin Nort Am 2001;30:61-79.

11. Ulick S, Gautier E, Vetter KK, Markello JR, Yaffe, Lowe Cu. An aldosterone biosynthetic defect in a salt-losing disorder. J Clin Endocrinol Metab 1964;24:669-672.

12. Bizzarri C, Pedicelli S, Cappa M, Cianfarani S. Water balance and 'salt wasting' in the first year of life: The role of aldosterone-signaling defects. Horm Res Paediatr 2016;86:143-153. Epub 2016 Sep 7

13. Martinerie L, Viengchareun S, Delezoide AL, Jaubert F, Sinico M, Prevot S, Boileau P, Meduri G, Lombès M. Low renal mineralocorticoid receptor expression at birth contributes to partial aldosterone resistance in neonates. Endocrinology 2009;150:4414-4424. Epub 2009 May 28

14. Wasniewska M, De Luca F, Valenzise M, Lombardo F, De Luca F. Aldosterone synthase deficiency type I with no documented homozygous mutations in the CYP11B2 gene. Eur J Endocrinol 2001;144:59-62.

15. Li N, Li J, Ding Y, Yu T, Shen Y, Fu Q, Shen Y, Huang X, Wang J. Novel mutations in the CYP11B2 gene causing aldosterone synthase deficiency. Mol Med Rep 2016;13;3127-3132. Epub 2016 Feb 18

16. Üstyol A, Atabek ME, Taylor N, Yeung MC, Chan AO. Corticosterone methyl oxidase deficiency type 1 with normokalemia in an infant. J Clin Res Pediatr Endocrinol 2016;8:356-359. Epub 2016 Apr 29

17. White PC. Aldosterone synthase deficiency and related disorders. Mol Cell Endocrinol 2004;217:81-87.

18. Lee PDK, Patterson BD, Hintz RL, Rosenfeld RG. Biochemical diagnosis and management of corticosterone methyl oxidase type II deficiency. J Clin Endocrinol Metab 1986;62:225-229.

19. Jessen CL, Christensen JH, Birkebæk NH, Rittig S. Homozygosity for a mutation in the CYP11B2 gene in an infant with congenital corticosterone methyl oxidase deficiency type II. Acta Paediatr 2012;101:519-525.

20. Haycock GB. The influence of sodium on growth in infancy. Pediatr Nephrol 1993;7:871-875.

21. Rösler A. The natural history of salt-wasting disorders of adrenal and renal origin. J Clin Endocrinol Metab 1984;59:689-700. 